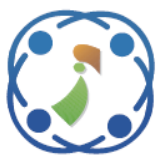

\title{
BER Analysis of Translation Invariant Wavelet Based Orthogonal Frequency Division Multiplexing System for 5G Wireless Communication Networks
}

\author{
Sumalatha Sunkara ${ }^{1 *}$ \\ Gollamandala Udaykiran Bhargava ${ }^{2}$ \\ ${ }^{1}$ Chamakura Malla Reddy Technical Campus, Hyderabad, Telangana, India \\ ${ }^{2}$ Mother Theresa Institute of Science and Technology, Sathupally, Telangana, India \\ *Email: sunkarasumalatha25@gmail.com
}

\begin{abstract}
A good orthogonality is provided by the wavelet -based orthogonality frequency division multiplexing (W-OFDM) and well reduction of bit error rate is gained with W-OFDM over conventional OFDM systems. Most importantly, it provides enhanced spectral efficiency since it does not require cyclic prefix (CP) which leads to the achievement of ultra-high data rates. Hence, this can be used in $5^{\text {th }}$ generation $(5 \mathrm{G})$ wireless communication networks. It is proposed to use the discrete wavelet transform (DWT) based OFDM in place of conventional discrete fourier transform (DFT) based OFDM in the applications of long term evaluation (LTE). However, discrete wavelet transforms (DWT) based OFDM system suffers from the down sampling, which mitigates the actual size of input bit streams and there by degradation in the system efficiency. To resolve this issue, and for enhancing further mitigation in bit error rate (BER) performance, a novel W-OFDM is proposed. Our proposed W-OFDM system utilizes translation invariant wavelet transform (TIWT) in place of DWT for enhanced spectral efficiency. In addition, fading channel environment is considered to disclose the effectiveness of proposed TIWT-OFDM system. Extensive experimental results show that the BER performance of proposed TIWT-OFDM is superior to the existing OFDM systems like DFT-OFDM and DWT-OFDM systems.
\end{abstract}

Keywords: Orthogonal frequency division multiplexing, Cyclic prefix, DFT-OFDM, 5G wireless networks, Discrete wavelet transform, W-OFDM, DWT-OFDM, Additive white gaussian noise, Translation invariant wavelet transform, Rayleigh fading channel, Bit error rate.

\section{Introduction}

Rapid enhancement of the technology has made it potential for the communication systems to transfer the data everywhere. But due to the assigned bandwidth limitation, it restricts the availability of bandwidth to the many users, which motivated the researchers to implement the bandwidth efficient transmission of data over a wireless communication system. Due to the frequency selective (FS) fading channels there is an occurrence of inter symbol interference (ISI), which in results degradation in the communication system channels performance which operates for higher data rates. Generally, the impairments like ISI, shadowing and fading caused due to the multi user accessibility. These impairments create unnecessary distortions in the communication system which leads to the system degradation and higher the BER values. To address this issue, multicarrier modulation (MCM) approach is presented, which transmits the input serial data stream as few parallel streams with low data rate [1-2]. Orthogonal frequency division multiplexing (OFDM) is a such technique which operates based on multi-carrier modulation approach. OFDM generates few parallel subcarriers by dividing the available spectrum and then each of them gets modulated by a low rate data stream at various carrier frequencies [3]. Fig. 1 disclosed that the DFT-based OFDM system which multiplexes the modulated signal using inverse fast fourier transform (IFFT) in the transmitter section later fast fourier transform (FFT) utilized in the receiver section that reduces the complexity at both 


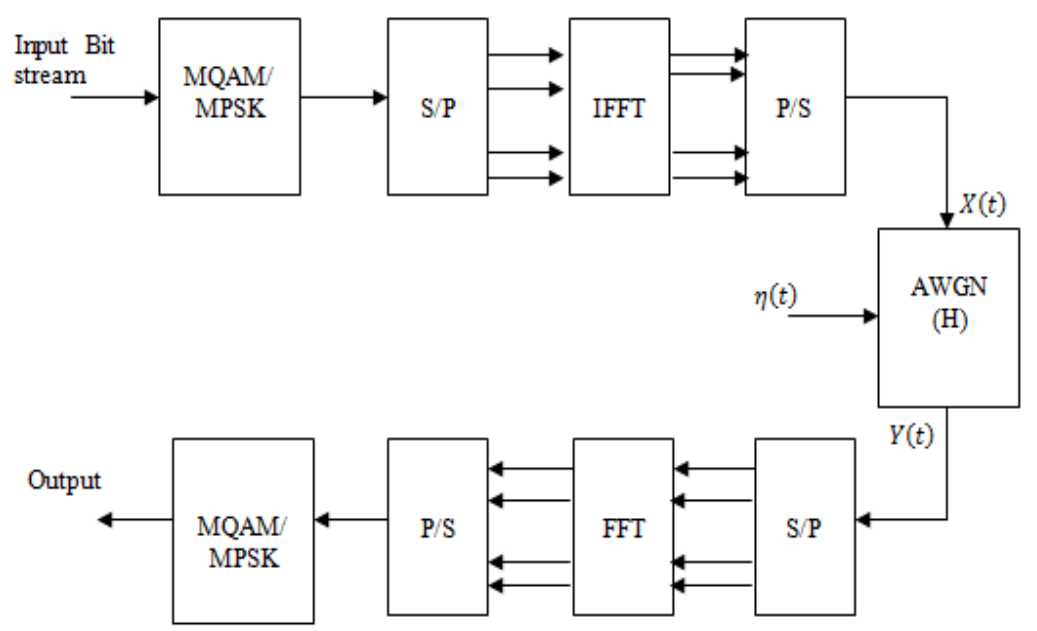

Figure. 1 DFT based OFDM system

transmitter and receiver end [4]. OFDM is constituted of a modulation and multiplexing combination. In OFDM, many independent signals will be generated by splitting the original data streams, each of which is modulated at a various frequency ranges and then these are multiplexed to produce an OFDM carrier. Due to the orthogonality nature of each subcarrier in OFDM, simultaneous transmission can be done without interference over the same bandwidth [4]. It makes the stronger highspeed data streams those are against to the impairments caused in radio channel environment.

OFDM is an effective approach to deal with the higher data rate transmission even under multipath fading environment. With the assistance of OFDM, parallel transmission of overlapped narrowband orthogonal subcarriers is done within the availability of transmission bandwidth, which in results efficient utilization of available spectrum. It also has the capability to fulfil this requirement for larger capacities and it is more economical and reliable to handle the processing power of digital signal processors [5]. All these advantages make OFDM a strong candidate for the $5^{\text {th }}$ generation wireless communication systems. Nowadays, it is utilized in various applications in the field of communication systems and telecommunication networks like IEEE 802.11 wireless standard, general switched telephone network (GSTN), cellular radios, digital audio, terrestrial digital video and high definition television (HDTV) broadcasting, modems with digital subscriber line (DSL) and asymmetric DSL (ADSL), high performance local area network (HIPERLAN) type I and type II.

Discrete wavelet transforms based OFDM (DWT-OFDM) [6], which is also an MCM technique, possesses almost all advantages and disadvantages of conventional (Fourier based)
OFDM. In this technique, the sub bandwidth division is obtained by using the inverse discrete wavelet-based transforms, whereas conventional OFDM uses IFFT [7]. Another main difference is that W-OFDM symbols overlap in both time and frequency domains, whereas OFDM symbols overlap only in frequency domain. Therefore, adding CP to the DWT-OFDM symbol frame does not have any effect on the bit error rate (BER) performance, as also shown in this work. One major advantage of DWT-OFDM compared to OFDM is that DWT-OFDM is more bandwidth efficient than OFDM [8-9]. For this reason, there are some practical applications such as those in [10-13]. Author in [14] presented a performance assessment of DFT-OFDM and DWT-OFDM in presence of high power amplifier (HPA) non-linearity. In [15], author has presented a DWT-IDWT based OFDM transmitter and receiver that achieved better performance in terms BER for AWGN channel. It has proven that all the wavelet performs better over the conventional IFFT-FFT implementation. Later they presented a comparative analysis on DWTOFDM and FFT-OFDM systems. Also, selection of mother wavelet is disclosed in this [16] with various wavelet families. In [17], DCT based OFDM system is presented and compared with the W-OFDM system which worked on $60 \mathrm{GHz}$ band of frequency. Author in [18] disclosed the comparison analysis of DFT-OFDM with DCT-OFDM under Rayleigh fading channel. Performance evaluation of DFTOFDM and DWT-OFDM with channel estimation techniques like least square (LS) and linear minimum mean square error (LMMSE) is presented in [19]. Further, they evaluated the performance of BER with various wavelets and the performance of mean square error (MSE) with LS and LMMSE estimators through block type pilot-based 
methodology. A replacement of discrete multiwavelet transforms (DMWT) based OFDM over conventional DFT-OFDM can reduce the interference level and enhances the spectral efficiency, which had demonstrated in [20]. It was also found that the DMWT-OFDM design achieved much lower BER performance and can be utilized as an alternative to the conventional DFT-OFDM. Another advancement of DWT-OFDM system is presented in [21], which disclosed the performance of BER in presence of carrier frequency offset (CFO) and Doppler-effect. Fading channels with exponential power delay profile is considered in [22], which also provided the performance of $\mathrm{W}$ OFDM and compared to that of DFT-OFDM. They considered Rayleigh channel conditions as a fading channel. In [23], multiple-input-multiple-output (MIMO) based OFDM system is presented. Further, they enhanced the MIMO for DWT based MIMOOFDM with several modulation and channel conditions. Author in [24] presented the similar DWT-OFDM system discussed above with the presence of CFO effect under AWGN channel environment. Recently, novel W-OFDM model is presented in [25], which is mainly intended to obtain a good orthogonality with much better spectral efficiency over conventional DFT-OFDM using different modulations.

However, most of the W-OFDM systems presented in the literature utilizes AWGN channel environment and they utilized discrete wavelet transform (DWT), which suffers from the decimation process while decomposing the input signal into approximation and detail coefficients, which reduces the original size of the input bit stream that results in loss of original data at the receiver end. To resolve this issue, another wavelet extension named as translation invariant wavelet transform (TIWT) is utilized in place of DWT. And to the best of our knowledge, none of the works in the open literature has given satisfactory BER performance results with DWT-OFDM and DFTOFDM in multipath Rayleigh fading channels.

The novel contributions of this work can be summarized as follows:

- Novel utilization of translation invariant wavelet transforms (TIWT) which is an extension for the discrete wavelet transform (DWT). This approach has not been used for OFDM applications before, according to the best knowledge of the authors.

- A fully new framework for W-OFDM system is proposed by utilizing translation invariant wavelet transform (TIWT) with fading channel environment and power delay profile.

- We mainly aimed at enhancing the spectral efficiency and mitigation of BER performance under fading conditions and power delay profile which was not focused in the past research work done in this field.

- To overcome the drawbacks of DFT-OFDM with AWGN [], DWT-OFDM with AWGN [24], DFT-OFDM with Rayleigh fading [18] and DWT-OFDM with Rayleigh fading [21], our present work aims at performing the enhanced version of discrete wavelet i.e., translation invariant wavelet which is discussed in section problem outline.

The rest of the paper is organized as follows: Section 2 provides a brief description of the problem outline. The proposed system model is described in Section 3. Section 4 explains the results and comparative evaluations against various works presented in the literature. Finally, Section 5 concludes the paper followed by references.

\section{Wavelet based OFDM system}

General OFDM system with inclusion of wavelet by replacing the FFT block provides an efficient spectral efficiency with reduced BER since the advancement of wavelets over FFT-OFDM shown in Fig. 1. A perfect reconstruction of a signal is possible by the DWT approach and it reduces the complexity of hardware since the DWT-OFDM doesn't need the cyclic prefix (CP), which leads to the mitigation of bandwidth wastage and transmission power thereby enhances the data rate transmission to the ultra-speed. It is an effective approach for analysing the signals in both spatial and spectral domain and found that the better orthogonality can be provided by the DWT since it has compact localization in both time and frequency domains. Due to the properties of high spectral restraint, DWT could combat the narrowband interference and can make the communication system more robust and effective against the intercarrier interference (ICI), which is a huge drawback of conventional FFT realization based OFDM. Due to these advancements, DWT has utilized in various fields like compression, computer vision, radar systems, astronomy, nuclear engineering, biomedical, acoustics, computer graphics and animation, seismology, satellite and remote sensing, fractals and pure mathematics. 


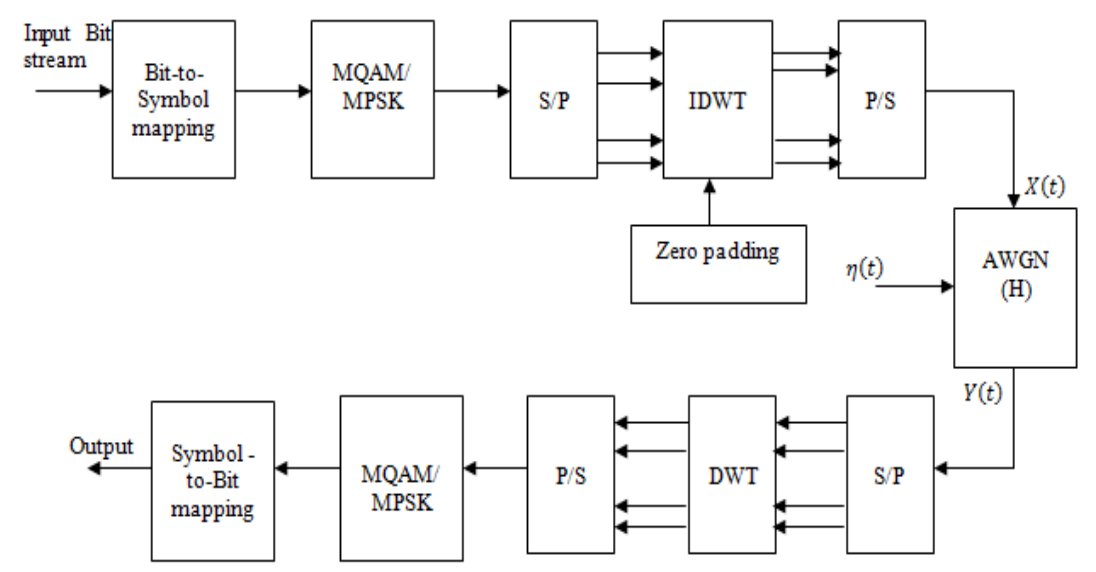

Figure. 2 DWT based OFDM system with AWGN channel

The structure of DWT-based OFDM system is demonstrated in Fig. 2. The modulated signal is transmitted using zero padding and vector transposing. DWT can decompose the input signal into number of sub bands which is known as approximated and detail coefficients as shown in Fig. 3 . These coefficients provide a far better analysis of a signal with both time and frequency scaling. Since DWT has many advantages such as flexibility, lesser sensitivity against interference and channel distortion with the optimal spectrum utilization, it has been proposed to design the advanced wireless communication systems like 5G networks and beneficial in various aspects such as modelling of channel, designing of transceivers data representation, source and channel coding, energy efficient networking and signal de-noising in wireless communication systems.

As shown in Fig. 3, approximated and detail coefficients of a source signal $x$ is computed by passing it via filter series like lowpass and high-pass filter banks. The input samples sent via a low-pass filter with impulse response $g$ leads to the convolution of the two:

$$
y[n]=(x * g)[n]=\sum_{k=-\infty}^{\infty} x[k] g[n-k]
$$

A simultaneous decomposition is done by utilizing the high-pass filter $h$ which produces the detail coefficients. It is vital that the both low-pass and high-pass filters should be related to each other, which can also call as quadrature mirror filter (QMF). Fig. 4 shows that the multi-level decomposition of DWT process. It demonstrated that the 3-level decomposition. In first level of decomposition approximated and detail coefficients is obtained after applying the down-sampling process. In second and third stage, the detail

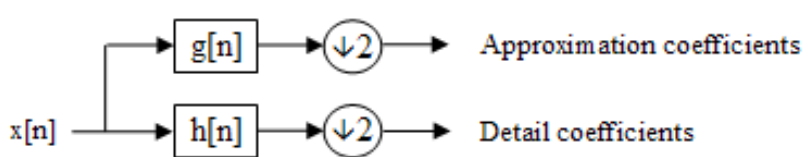

Figure. 3 Filter analysis block diagram of wavelet

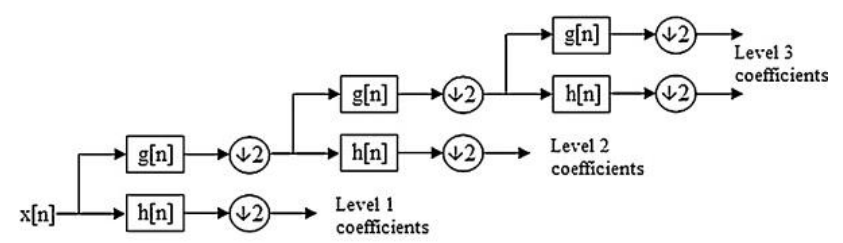

Figure. 4 Multi level decomposition of DWT

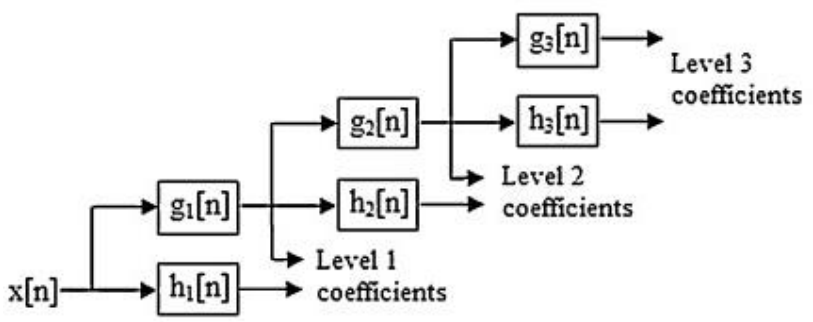

Figure. 5 Multi level decomposition of proposed TIWT

coefficients from the level one and two ignored respectively and only approximated coefficients further decomposed into approximated and detail coefficients.

However, major limitations of DWT are shift variance deficiency which occurs due to the down sampling or sub sampling nature of DWT and poor directional selectivity. Huge differentiations in wavelet coefficients caused by the minor shifts of input signal which in results the differences in energy distribution between the coefficients of wavelet at different scales. In addition, this also leads to the improper reconstruction of an input signal. Therefore, one must design a wavelet with the elimination of the down-sampling and should enhance the spectral efficiency further for ultra- 
speed communication systems. One possible solution for this is shift invariant DWT without having the down-sampling step in the decomposition structure. Here, authors introduced a translation invariant wavelet transform (TIWT) for enhancing the spectral efficiency further and mitigating the BER. The decomposition tree of TIWT process is shown in Fig. 5, where there is no down-sampling factor. Another recognizing feature of TIWT is redundancy that introduces over-complete frame expansion, which enhances the robustness against additive noise as compared to the orthogonal expansion. Hence, due to this frame expansion feature, the outcome coefficients from the decomposition of TIWT has lesser noisy effected over conventional DWT.

\section{System model}

This section describes the proposed model frame work in detail. Here, wavelet based OFDM system is presented to enhance the communication system efficiency in terms of lower BER values and higher data rates for $5 \mathrm{G}$ network applications. The utilization of translation invariant wavelet transform improves the spectral efficiency of OFDM system by reducing the hardware expenditure. It doesn't need cyclic prefix block, which is an essential block in conventional DFT based OFDM system. The discrete time OFDM symbol at the transmitter can be expressed as

$$
x[n]=\frac{1}{\sqrt{N}} \sum_{k=0}^{N-1} X(k) e^{\frac{j 2 \pi n k}{N}}, n=0,1,2, \ldots \ldots, N-1
$$

where $\mathrm{N}$ is total numbers of subcarriers and $\mathrm{X}$ (k) denotes the modulated data symbol transmitted on $k^{\text {th }}$ subcarrier.

Fig. 6 shows that the proposed TIWT-based OFDM system with Rayleigh fading channel environment. Let $X(n)$ be the input signal and $Y(n)$ be its reconstructed version. $a[-k]$ and $d[-k]$ are lowpass and high pass analysis filters while $a[k]$ and $d[k]$ are corresponding lowpass and high pass synthesis filters. $A_{j}$ and $D_{j}$ are output coefficients at level j. TIWT avoids down-sampling and upsampling of coefficients. Analysis and synthesis filter bank for TIWT is shown Fig. 7.

For DWT, analysis and synthesis can be expressed as,

$$
A_{j}[k]=\left(A_{j+1}[k] * a_{j}[-k]\right) \downarrow 2
$$

and

$$
D_{j}[k]=\left(A_{j+1}[k] * d_{j}[-k]\right) \downarrow 2
$$

Where $*$ denotes convolution and $\downarrow 2$ represents down sampling by a factor of 2 .

Synthesis can be done by using,

$$
A_{j+1}[k]=\left(A_{j}[k] \uparrow 2\right) * a[k]+\left(D_{j} \uparrow 2\right)[k] * d[k]
$$

Where $\uparrow 2$ denotes up sampling by a factor 2 . Now, this down and up samplings operation in DWT is eliminated in TIWT and number of output coefficients doubles that of input. Filters are up sampled to fit to the length of growing data. They can be expressed as

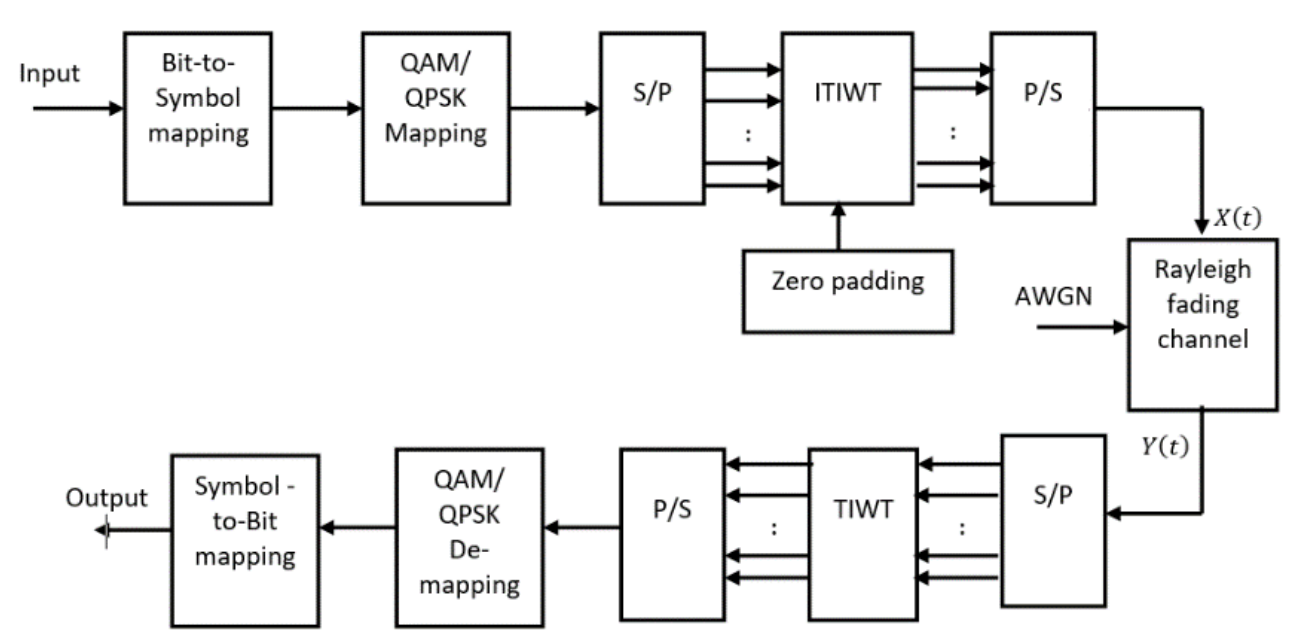

Figure. 6 Proposed TIWT-OFDM system 


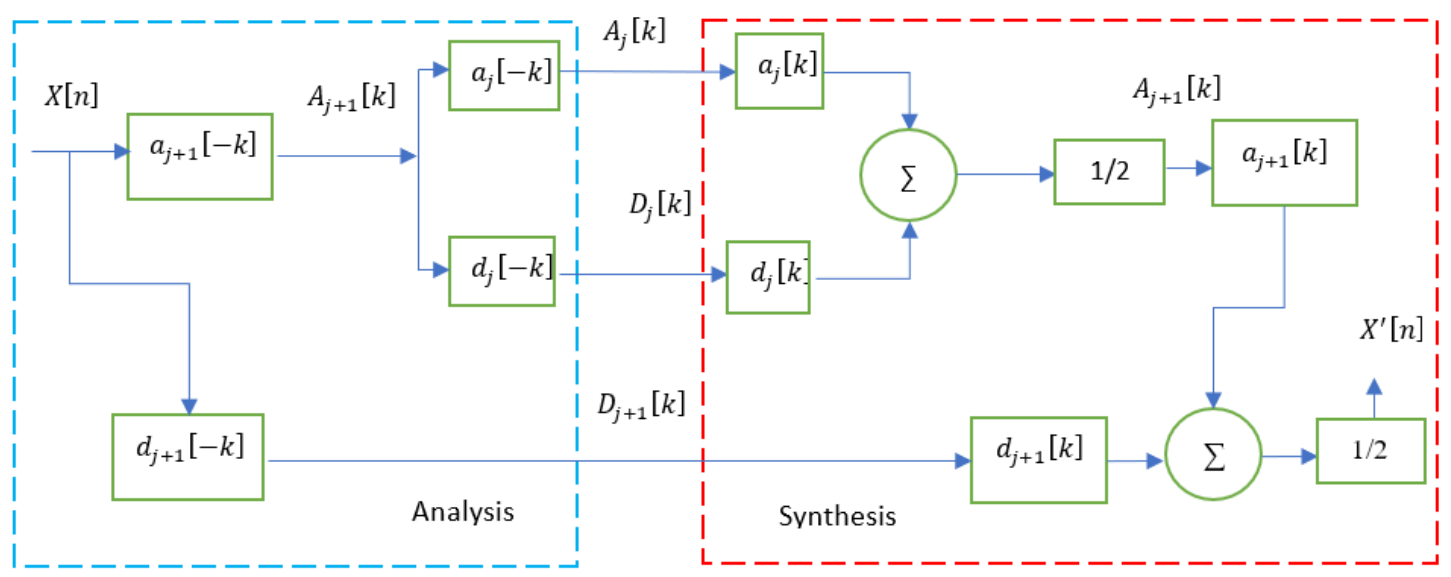

Figure. 7 Structure of TIWT

$a_{j}[K]=a_{j+1}[K] \uparrow 2$

$d_{j}[K]=d_{j+1}[K] \uparrow 2$

It results in overcomplete representation. TIWT analysis can be mathematically expressed as,

$$
\begin{aligned}
& A_{j}[k]=\left(A_{j+1}[k] * a_{j}[-k]\right) \\
& D_{j}[k]=\left(A_{j+1}[k] * d_{j}[-k]\right)
\end{aligned}
$$

Synthesis can be expressed as,

$$
A_{j+1}[k]=\frac{1}{2}\left(A_{j}[k] * a_{j}[k]+D_{j}[k] * d_{j}[k]\right)
$$

TIWT maintains uniform sampling rate in time domain.

\section{Results and discussion}

This section describes the experimental analysis of proposed TIWT-OFDM system under Rayleigh fading channel. All the simulation parameters are shown in Table 1. Fig. 8 shown that the performance of proposed model BER with comparison to the conventional DFT-OFDM [12] and DWT-OFDM [24] with AWGN channel.

It has disclosed that the enhancement of BER mitigation while increasing the signal-to-noise ratio (SNR) values. At a value of $10 \mathrm{~dB}$ SNR, the proposed TIWT-OFDM system achieved a BER value of around $10^{-3}$, where the conventional DFTOFDM got $10^{-0.5}$ and DWT-OFDM got $10^{-1}$ at the same SNR range. It is almost three times higher than our proposed OFDM system.
Table 1. Simulation parameters

\begin{tabular}{|c|c|c|}
\hline Symbol & Parameter & Value \\
\hline$N$ & $\begin{array}{c}\text { Number of sub } \\
\text { carriers }\end{array}$ & $32,64,128, \ldots, N$ \\
\hline$M$ & Constellation points & $4,8, \ldots, M$ \\
\hline$k$ & $\begin{array}{c}\text { Number of bits per } \\
\text { symbol }\end{array}$ & $\log _{2} M$ \\
\hline$\xi$ & Over sampling rate & 4 \\
\hline$n$ & Number of bits & $10^{6}$ \\
\hline$H$ & Channel environment & $\begin{array}{c}\text { AWGN, } \\
\text { Rayleigh and } \\
\text { Rician }\end{array}$ \\
\hline$W$ & Type of wavelet & Daubieches \\
\hline
\end{tabular}

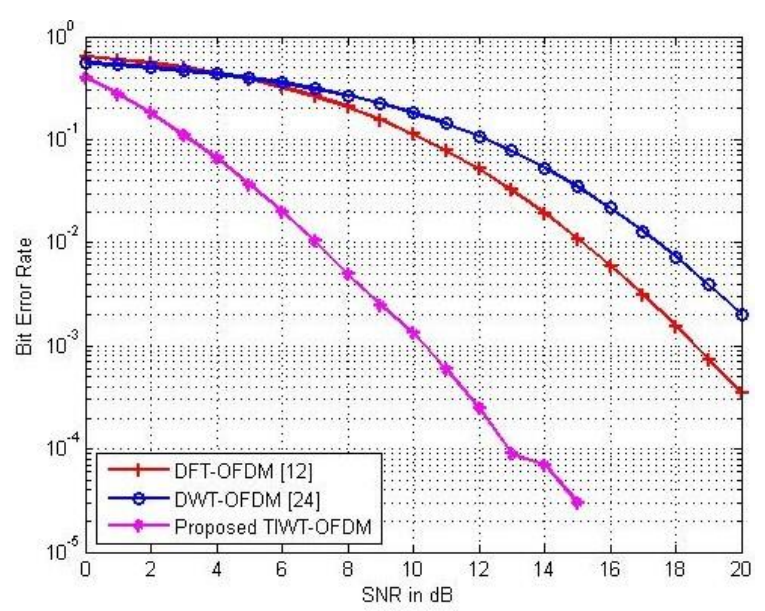

Figure. 8 BER performance of proposed TIWT-OFDM and conventional OFDM systems with AWGN channel

Table 2. Simulation parameters of Rayleigh fading channel

\begin{tabular}{|c|c|}
\hline Parameter & Value \\
\hline Symbol rate & $20 \times 10^{6}$ \\
\hline Sampling time & $1 /$ symbol rate \\
\hline Tap delay in sec & {$\left[\begin{array}{lll}0.0 & 0.4 & 0.9\end{array}\right] \times 10^{-6}$} \\
\hline $\begin{array}{c}\text { Power delay profile in } \\
\text { dB }\end{array}$ & {$\left[\begin{array}{lll}0 & -5 & -10\end{array}\right]$} \\
\hline $\begin{array}{c}\text { Maximum doppler } \\
\text { frequency }\end{array}$ & 100 \\
\hline
\end{tabular}




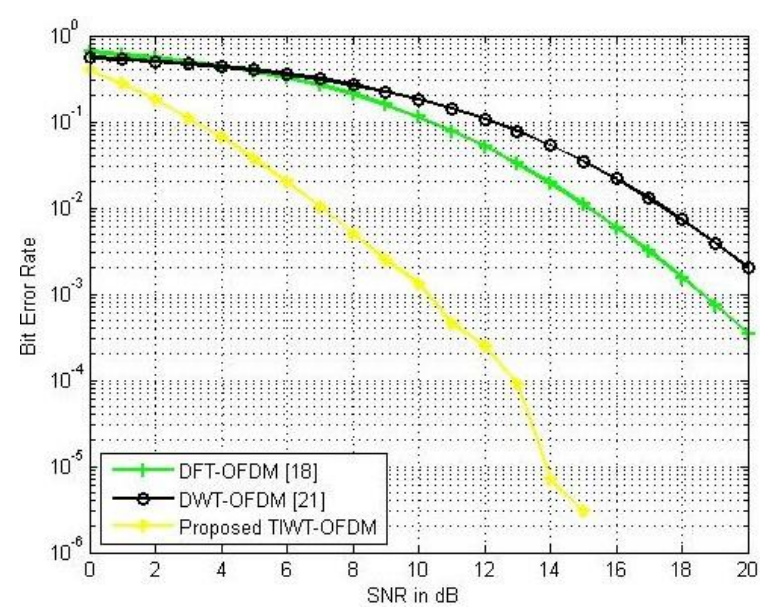

Figure. 9 BER performance of proposed TIWT-OFDM and conventional OFDM systems with Rayleigh channel

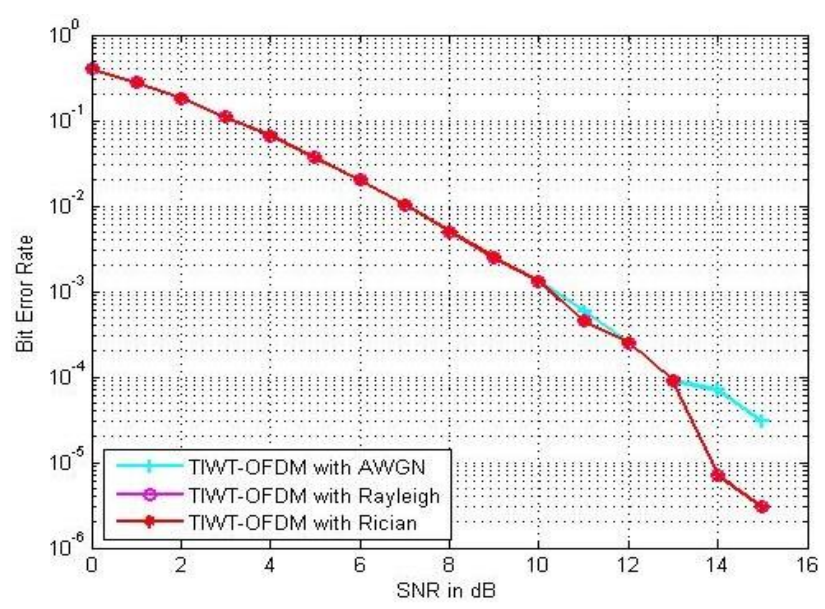

Figure. 10 BER analysis of proposed model with AWGN, Rayleigh fading and Rician channels

However, the performance of proposed TIWTOFDM is further enhanced under the fading channel environment like Rayleigh fading channel, this shows the effectiveness of our proposed system model also motivates us for utilization of proposed methodology in $5^{\text {th }}$ generation applications. Table 2 shown that the simulation parameters utilized in generation of Rayleigh fading channel environment. Fig. 9 demonstrates that the BER performance with Rayleigh fading channel. There is not much changes in the performance of conventional DFT-OFDM [18] and DWT-OFDM [21] with the Rayleigh fading channel where as our proposed model got further mitigated performance of BER.

Fig. 10 disclosed that the performance analysis BER with AWGN channel, Rayleigh and Rician fading channel environments. It is given that the BER curve of proposed TIWT-OFDM system under Rayleigh fading and Rician is almost similar ones. Selection of simulation parameters for generating the Rayleigh fading channel is provided in Table 2. For Rician generation also similar values have been utilized and additionally a value of 0.1 as a Rician factor in linear scale.

\section{Conclusions}

Here a novel W-OFDM system is presented for enhancing the efficiency of wireless communication network by mitigating the BER performance. A new version of wavelet named translation invariant wavelet is introduced to supress the limitation of conventional discrete wavelet, which reduces the actual size of data stream with the nature of decimation process. Utilization of TIWT-OFDM with AWGN channel provided an excellent spectral efficiency with the degradation in BER performance over the conventional OFDM systems like DFTOFDM and DWT-OFDM. Additionally, author considered fading channel environment conditions like Rayleigh channel and compared the BER performance with the DFT-OFDM and DWTOFDM there by achieved ultra-high data rates for $5 \mathrm{G}$ wireless networks. Further, a simple comparative analysis of proposed TIWT-OFDM with various channel environments like AWGN, Rayleigh and Rician also provided for better BER analysis. In future, more better transformations with optimal algorithms can be implemented to mitigate the BER further to gain the massive data rates and can be utilized for enhanced generations of wireless communication networks.

\section{References}

[1] J. A. C. Bingham, "Multicarrier modulation for data transmission: An idea whose time has come", IEEE Communication Magazines, Vol. 28, No. 5, pp. 5-14, 1990.

[2] P. Gotz, U. Jorn, K. Werner, and Z Georg, "A comparison of various MCM schemes", In: Proc. of the $5^{\text {th }}$ International Workshop on OFDM, pp. 20-1 - 20-5, 2000.

[3] L. Jun, T. T. Thiang, F. Adachi, and H. C. Li, "BER performance of OFDM-MDPSK system in frequency selective Rician fading and diversity reception", IEEE Transactions on Vehicular Technology, Vol. 49, No. 4, pp. 12161225, 2000.

[4] D. N. Muhammad, N. Hans, and H. Thomas, "On Performance Limits of DFT Spread OFDM Systems", In: Proc. of IST Mobile and Wireless Communications Summit, 2007.

[5] T. Hwang, C. Yang, W. Gang, S. Li, and G. Y. Li, "OFDM and Its Wireless Applications: A Survey", IEEE Transactions on Vehicular Technology, Vol. 58, No. 4, pp. 1673-1694, 2009. 
[6] R.W. Klein, M.A. Temple, R.A. Raines, and R.L. Claypoole Jr., "Interference Avoidance Communications using Wavelet Domain Transformation Techniques", IEEE Electronic Letters, Vol. 37, No. 15, pp. 987-989, 2001.

[7] D. Guptha, V.B. Vats, and K.K. Garg, "Performance Analysis of DFT-OFDM, DCTOFDM and DWT-OFDM Systems in AWGN Channel", In: Proc. of International Conference on Wireless and Mobile Communications, pp. 214-216, 2008.

[8] M. B. Veena and M.N.S. Swamy, "Performance analysis of DWT based OFDM over FFT based OFDM and implementing on FPGA", International Journal of VLSI design \& Communication Systems, Vol.2, No. 3, pp. 119130, 2011.

[9] B. Gupta, G. Gupta, and D. S. Saini, "BER Performance Improvement in OFDM System with ZFE and MMSE Equalizers", In: Proc. of International Conference on Electronics Computer Technology, pp. 193-197, April 2011.

[10] K. Oguz and K. Volkan, "Alamouti coded wavelet based OFDM for multipath fading channels", In: Proc. of Wireless Telecommunications Symposium, pp.1-5, 2009.

[11] R. Mika and T. Olav, "LTE, the radio technology path toward 4G", Computer communications, Vol. 33, No. 16, pp. 18941906, 2010.

[12] U. Kumar, C. Ibars, A. Bhorkar, and H. Jung, "A Waveform for 5G: Guard Interval DFT-sOFDM", In: Proc. of IEEE Globecom Workshops, 2015.

[13] A. Sahin, E. Bala, R. Yang, and R. L. Olesen, "DFT-Spread OFDM with Frequency Domain Reference Symbols", In: Proc. of IEEE Conference on Global Communications, Singapore, 2017.

[14] A. Kaini, G. Baghersalimi, and B. Zanj, "Performance assessment of DFT-OFDM and DWT-OFDM systems in the presence of the HPA Nonlinearity", In: Proc. of International Conference on Telecommunications, pp. 273278, 2011.

[15] R. Bodhe, S. Narkhede, and S. Joshi, "Design of Simulink Model for OFDM and Comparison of FFT-OFDM and DWT-OFDM", International Journal of Engineering Science and Technology, Vol. 4, No. 5, pp. 1914-1924, 2012.

[16] R. Bodhe, S. Joshi, and S. Narkhede, "Performance Comparison of FFT and DWT based OFDM and Selection of Mother Wavelet for OFDM", International Journal of Computer
Science and Information Technologies, Vol. 3, No. 3, pp. 3393-3397, 2012.

[17] A. Deshmukh and S. Bodhe, "Comparison of DCT and Wavelet Based OFDM System Working in $60 \mathrm{GHz}$ Band", International Journal of Advancements in Technology, Vol. 3, No. 2, pp. 74-83, 2012.

[18] L. Patidar and A. Parikh, "BER Comparison of DCT-based OFDM and FFT-based OFDM using BPSK Modulation over AWGN and Multipath Rayleigh Fading Channel", International Journal of Computer Applications, Vol. 31, No. 10, pp. 38-41, 2011.

[19] G. M. Kumar and S. Tiwari, "Performance evaluation of conventional and wavelet based OFDM system", International Journal of Electronics and Communications, Vol. 67, No. 4, pp. 348-354, 2013.

[20] A. H. Kattoush, W.A. Mahmoud, and S. Nihad, "The performance of multi wavelets based OFDM system under different channel conditions”, Digital signal processing, Vol. 20, No. 2, pp. 472- 482, 2010.

[21] N. Hariprasad and G. Sundari, "Performance comparison of DWT OFDM and FFT OFDM in presence of CFO and Doppler effect", In: Proc. of International Conference on Control, Instrumentation, Communication and Computational Technologies, pp. 567-570, 2014.

[22] V. Kumbasar and O. Kucur, "Performance comparison of wavelet based and conventional OFDM systems in multipath Rayleigh fading channels", Digital Signal Processing, Vol. 22, No. 5, pp. 841-846, 2012.

[23] A. Bouhlel, A. Sakly, and N. Mansouri, "Performance Comparison of DWT based MIMO OFDM and FFT based MIMO OFDM", In: Proc. of International Conference on Advanced Wireless, Information, and Communication Technologies, Vol. 73, pp. 266-273, 2015.

[24] A. Jhingan and L. Kansal, "Performance Evaluation for Wavelet based OFDM system effected by CFO over Rayleigh Channel", Indian Journal of Science and Technology, Vol. 9, No. 5, pp. 1-6, 2016.

[25] M. Praveen, E. Adinarayana, and V. S. R. Kumari, "Implementation of OFDM communication model for achieving the improved BER using DWT-OFDM", International Journal of Engineering and Computer Science, Vol. 6, No. 1, pp. 19511959, 2017. 